\title{
A New Modified CMM Modular Exponentiation Algorithm
}

\author{
Abdalhossein Rezai \\ Semnan University, Semnan, Iran
}

\author{
Parviz Keshavarzi \\ Semnan University, Semnan, Iran
}

\begin{abstract}
Modular exponentiation is fundamental operation in the many cryptosystem such as RSA. This operation is implemented by repeating modular multiplication which is time consuming for large operands. This paper presents a new modified Montgomery modular multiplication algorithm based on multiple bit scan-multiple bit shift technique, sliding window method and signed-digit representation. This new algorithm skips over zero digit multiplication and the following required addition. Then it shifts the partial results by using Barrel shifter in only one cycle instead of several cycles. In addition, we proposed new modular exponentiation algorithm based on this new modular multiplication algorithm and common-multiplicand-multiplication method. In this new algorithm, the common part of modular multiplication is computed once rather than several times. So the security of the cryptosystem which used this new algorithm increased considerably. The analysis results show that the number of multiplication steps in the proposed exponentiation algorithm is reduced on average at about $71.8 \%-89.2 \%$, $66.2 \%-87.1 \%, 15.4 \%-67.6 \%$ and $53.8 \%-82.3 \%$ in compare with Dusse-Kaliski's algorithm, Ha-Moon's algorithm, Wu et al.'s algorithm and Wu's algorithm respectively for $d=3-10$.
\end{abstract}

\section{Introduction}

The fundamental operation of the many public-key cryptosystem (PKC) such as RSA is the large integers modular exponentiation which is implemented by repeating modular multiplication [1][2][3]. So the efficiency of the many PKC is primarily determined by the efficiency of the modular multiplication algorithm [4][5][6].

Montgomery modular multiplication (M3) algorithm [7] is an efficient algorithm for modular multiplication because it avoids division by the modulus [8][9].There are many research efforts in order to speed up the performance of the Montgomery modular multiplication algorithm such as high-radix design [10][11][12], scalable design [8][11] [12], parallel calculation quotient and partial result [3] and signed-digit recoding [9][13][14].

$\mathrm{Ha}$ and Moon in [15] proposed that the common part of modular multiplication in modular exponentiation can be computed once rather than twice and called it common-multiplicand multiplication (CMM) method. Wu et al. in [16] proposed using canonical recoding technique in order to recode the exponent. So the probability of the nonzero digit is reduced. Therefore the computational complexity of the modular exponentiation is decreased. In [16] CMM method in [15] is used in multiplication phase. $\mathrm{Wu}$ in [17] proposed divide the signed-digit exponent into three equal lengths and use of CMM technique in order to compute common part of multiplications, once rather than several times.

In this paper, a new Montgomery modular multiplication algorithm based on constant length nonzero (CLNZ) sliding window method, multiple bit scan, multiple bit shift and signed-digit technique is presented. This new algorithm is an improvement of the adaptive m-ary multiplication method [13]. In addition we proposed using this new modular multiplication in order to speed up the modular exponentiation algorithm.

The rest of this paper is organized as follows: section 2 describes the background of the proposed algorithms. The proposed modular multiplication algorithm and its application in improved CMM-MSD Montgomery modular exponentiation algorithm is presented in section 3. In section 4 security of the cryptosystem which used proposed algorithm is described. Section 5 evaluates the proposed algorithms. Finally conclusion is given in section 6 .

\section{Background}

This section outlines the Montgomery modular multiplication algorithm, the adaptive m-ary canonical recoding multiplication method and the CMM method to the Montgomery exponentiation algorithm.

\subsection{The Montgomery modular multiplication algorithm}

Montgomery modular multiplication (M3) algorithm which first introduced in [7], speeds up the modular multiplication and modular exponentiation algorithm by replacing the trial division by the modulus with a simple right shift [15][17]. Algorithm 1 shows the radix-2 Montgomery modular multiplication algorithm:

Algorithm 1: The radix-2
multiplication algorithm




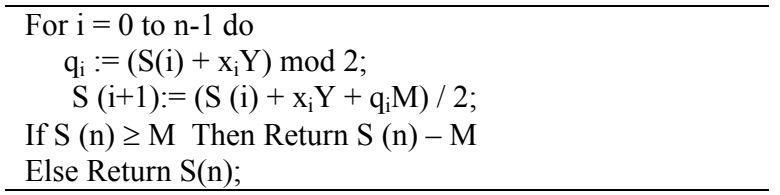

This algorithm computes $\mathrm{S}(\mathrm{n})=\mathrm{XY} 2^{-\mathrm{n}} \bmod \mathrm{M}$ in $\mathrm{n}$ - loop iterations. So it is time-consume operation.

\subsection{The adaptive $m$-ary canonical recoding multiplication method}

The m-ary segmentation and canonical recoding are two well-known methods in order to reduce the total number of the additions in multiplication and to reduce the total number of multiplications in exponentiation operation. The m-ary segmentation (radix-m) multiplication algorithm utilizes segmentation and pre-computation in order to reduce the number of addition $[5[6][10][13]$. Since the probability of a word of length $d$ being zero is $2^{-\mathrm{d}}$, longer words have smaller zero word probabilities. For increase efficiency of the occurrence probability, Koc and Hung in [13] proposed an adaptive m-ary method which allows zero words are variable lengths and improve zero word probability while using relatively long words in the segmentation process.

According to [13][14], in computing $\mathrm{P}=\mathrm{XY}$, we may skip additions whenever the corresponding bit of the multiplier is zero. Since the average number of nonzero bits in $n$-bit binary number is $n / 2$, the binary multiplication algorithm requires $n / 2$ addition operations on average. Since the average number of nonzero bits in n-bit canonical recoding representation is $\mathrm{n} / 3$, the multiplication algorithm with canonical recoding multiplier requires $n / 3$ addition operations on average [14]. Koc and Hung in [13] proposed the combination of the adaptive m-ary segmentation algorithm and the canonical recoding algorithm in order to obtain the adaptive m-ary segmentation canonical recoding multiplication algorithm which is shown in algorithm 2.

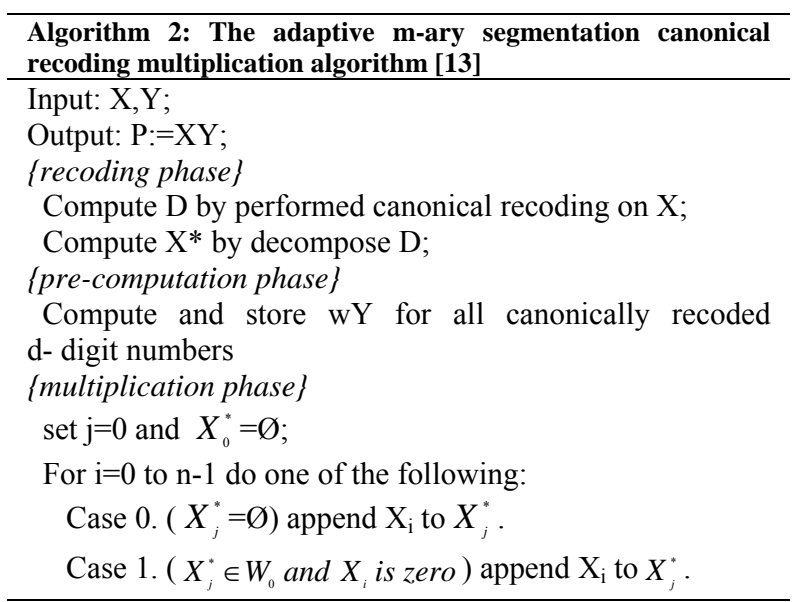

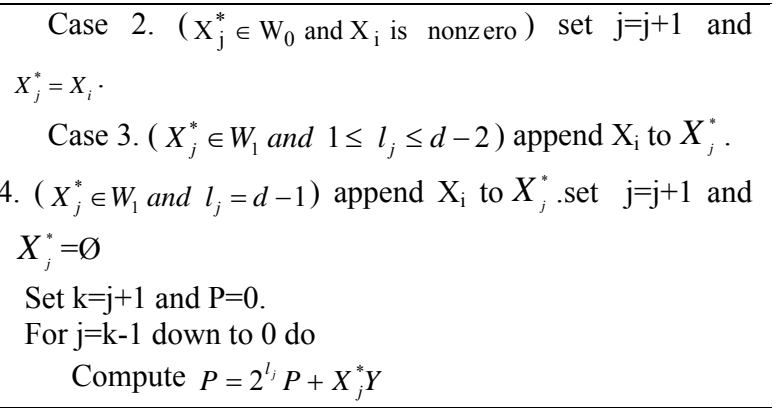

In this algorithm, $l_{j}$ denotes the length of $X_{j}^{*}$. Also the probability of the zero bits is increased by using canonical recoding technique. Also the total computation time is reduced by using m-ary segmentation.

\subsection{The CMM method for the Montgomery exponent algorithm}

As modular exponentiation (ME) consists of series of modular multiplications, the performance of the ME operation is determined by the efficiency of the implementation of the modular multiplication [1][5][17]. The Montgomery modular exponentiation algorithm is shown in algorithm 3 :

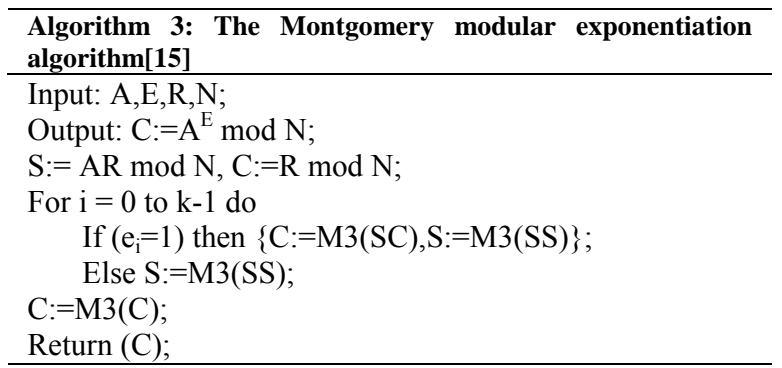

In algorithm 3, when the exponent bit is not zero (i.e. $\mathrm{e}_{\mathrm{i}}=1$ ), both M3(SC) and M3(SS), are performed. Ha and Moon proposed the common part in M3(SC) and M3(SS) can be computed once rather than twice [15]. They show that the computation of M3(SC) can be modified as following:

$$
\begin{aligned}
\mathrm{M} 3(\mathrm{SC}) & =\mathrm{SCR}^{-1} \bmod \mathrm{N}= \\
& \left.\mathrm{S}\left(\mathrm{C}_{\mathrm{n}-1} \mathrm{~b}^{\mathrm{n}-1}+\mathrm{C}_{\mathrm{n}-2} \mathrm{~b}^{\mathrm{n}-2}+\ldots+\mathrm{C}_{0} \mathrm{~b}^{0}\right) \mathrm{b}^{\mathrm{m}-\mathrm{n}}\right) \mathrm{b}^{-\mathrm{m}} \bmod \mathrm{N} \\
& =\left(\mathrm{C}_{\mathrm{n}-1}\left(\mathrm{Sb}^{\mathrm{m}-1} \bmod \mathrm{N}\right)+\mathrm{C}_{\mathrm{n}-2}\left(\mathrm{Sb}^{\mathrm{m}-2} \bmod \mathrm{N}\right)+\ldots\right. \\
& +\mathrm{C}_{0}\left(\mathrm{Sb}^{\mathrm{m}-\mathrm{n}} \bmod \mathrm{N}\right) \mathrm{b}^{-\mathrm{m}} \bmod \mathrm{N} .
\end{aligned}
$$

Where $b^{-n}=R^{-1} \bmod N$. The memory space and computation time depend on the value of $\mathrm{m}$. The optimal value of $\mathrm{m}$ is 2 [15]. For this value the $\mathrm{M} 3(\mathrm{SC})$ is represented as following:

$$
\begin{aligned}
\mathrm{M} 3(\mathrm{SC})= & \left(\mathrm{C}_{\mathrm{n}-1} \mathrm{~S}+\left(\mathrm{C}_{\mathrm{n}-2} \mathrm{~S}+\mathrm{C}_{\mathrm{n}-3}\left(\mathrm{Sb}^{-1} \bmod \mathrm{N}\right)+\ldots\right.\right. \\
& \left.+\mathrm{C}_{0}\left(\mathrm{Sb}^{2-\mathrm{n}} \bmod \mathrm{N}\right) \mathrm{b}^{-1}\right) \mathrm{b}^{-1} \bmod \mathrm{N} .
\end{aligned}
$$

Similarly, the M3(SS) is presented as following:

$$
\begin{aligned}
\mathrm{M} 3(\mathrm{SS})= & \left(\mathrm{S}_{\mathrm{n}-1} \mathrm{~S}+\left(\mathrm{S}_{\mathrm{n}-2} \mathrm{~S}+\mathrm{S}_{\mathrm{n}-3}\left(\mathrm{Sb}^{-1} \bmod \mathrm{N}\right)+\ldots\right.\right. \\
& \left.+\mathrm{S}_{0}\left(\mathrm{Sb}^{2-\mathrm{n}} \bmod \mathrm{N}\right) \mathrm{b}^{-1}\right) \mathrm{b}^{-1} \bmod \mathrm{N} .
\end{aligned}
$$


So, $\mathrm{Sb}^{-\mathrm{j}} \bmod \mathrm{N}$ for $1 \leq \mathrm{j} \leq \mathrm{n}-2$ is common to both $\mathrm{M} 3(\mathrm{SC})$ and M3(SS). Also $\mathrm{Sb}^{-\mathrm{j}}$ is computed by using the previous result, $\mathrm{T}=\mathrm{Sb}^{-\mathrm{j}+1} \bmod \mathrm{N}$, which is $\mathrm{Sb}^{-\mathrm{j}}$ $\bmod \mathrm{N}=\mathrm{Tb}^{-1} \bmod \mathrm{N}$. In this method, we can alternatively compute $\mathrm{Sb}^{-1}, \mathrm{C}_{\mathrm{n}-3} \mathrm{Sb}^{-1}, \mathrm{~S}_{\mathrm{n}-3} \mathrm{Sb}^{-1}, \mathrm{Sb}^{-2}$ and so on. Therefore the memory space and computation time are reduced considerably.

$\mathrm{Wu}$ et al. in [16] improved the CMM Montgomery exponentiation algorithm by using canonical recoding technique in order to reduce the Hamming weight of the exponent. The Wu et al.'s exponentiation algorithm is shown in algorithm 4 .

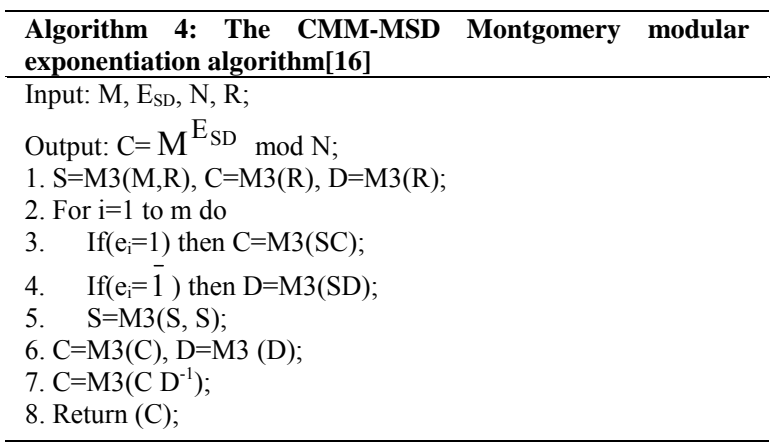

In this algorithm, the exponent $\mathrm{E}$ is recoding by using canonical recoding technique separately. So the probability of the nonzero digit in exponent is reduced. Also the modular multiplication required in modular exponentiation algorithm is reduced considerably. Therefore the speed of the modular exponentiation algorithm increases considerably.

Another attempt in order to speed up the performance of modular exponentiation algorithm based on this idea is the improved CMM-MSD Montgomery algorithm [17] which is shown in algorithm 5:

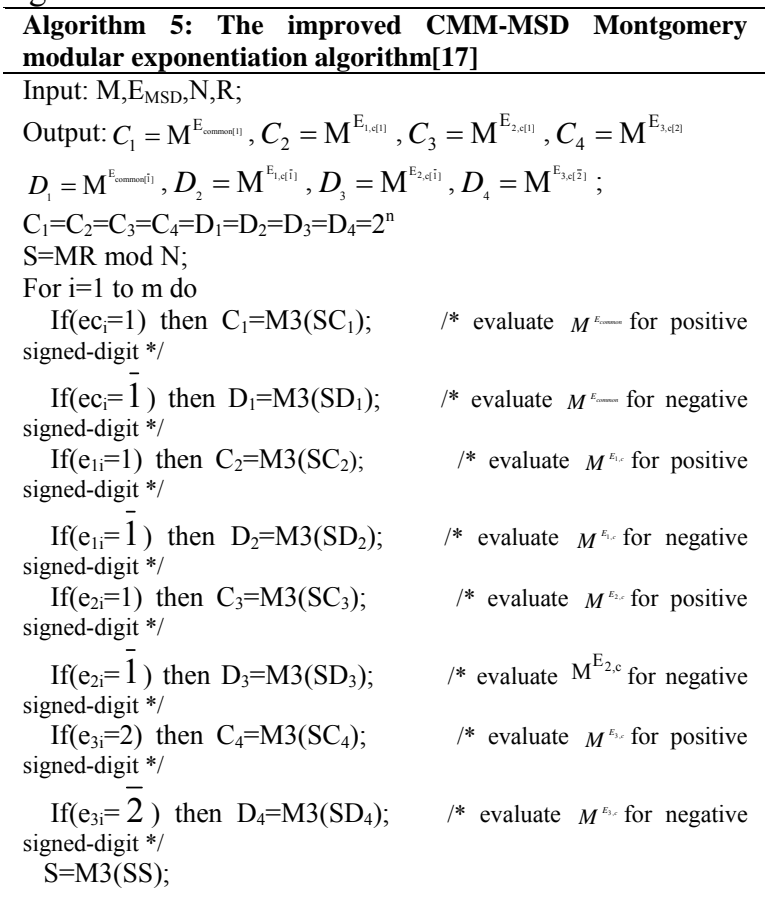

In algorithm 5, the exponent $\mathrm{E}_{\mathrm{MSD}}$ is divided into three equal lengths as $E_{1}, E_{2}$ and $E_{3}$ and the operation results of positive digits put in the registers $C_{i}, 1 \leq i \leq 3$ and the operation results of the negative digits put in the registers $D_{i}, 1 \leq i \leq 3$. The $C_{i}$ and $D_{i}$ are used in order to store the operation results in the decomposition segment of $E_{i}$ of minimal signed-digit exponent $\mathrm{E}_{\mathrm{MSD}}$. In addition in this algorithm by using CMM method, the common part of six multiplication can be computed just one. In this algorithm the exponentiation operation $\mathrm{M}^{\mathrm{E}}$ can be depicted as (1).

$$
M^{E}=M^{E_{1}\left\|E_{2}\right\| E_{3}}=M^{E_{1} \cdot 2^{n}} M^{E_{2}} \cdot 2^{2 n}{ }^{E_{3}}
$$

\section{The proposed CMM-MSD Montgomery exponentiation algorithm}

In serial-parallel multiplication, partial result shifts one bit per iteration. Also multiplication by zero bit results in zero, but this multiplication by zero is performed and implemented per iteration. In this paper, we proposed a new modified Montgomery modular multiplication by recoding and then by partitioning the multiplier. This performs multiplication by zero partition with any length in only one-cycle instead of several cycles. The proposed modular multiplication algorithm is shown in algorithm 6.

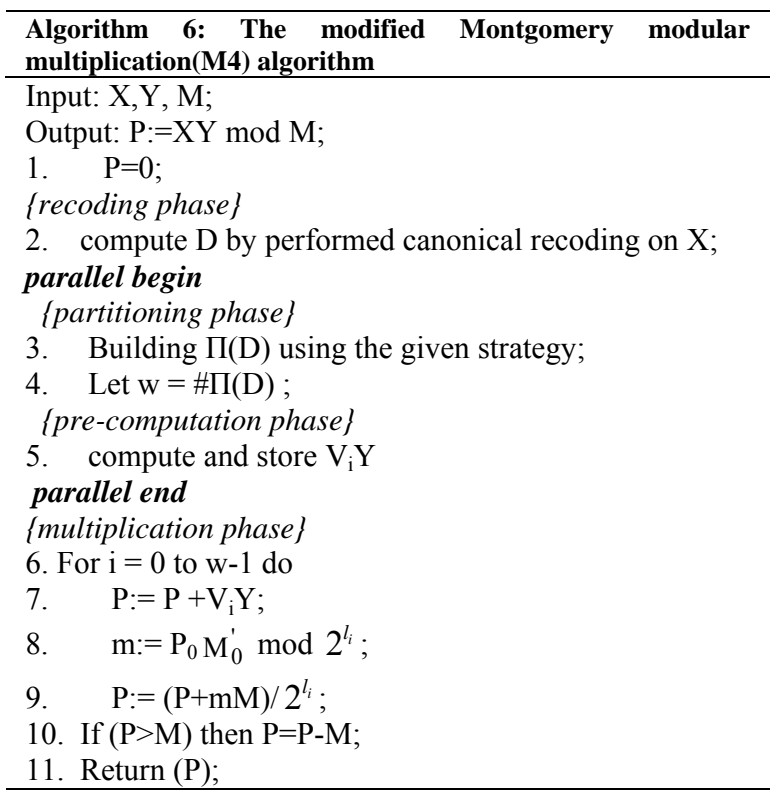

In this algorithm, $l_{i}$ is the length (i.e. the number of bits) of ith partition, \#П(D) is the number of partitioning in the multiplier and $\mathrm{V}_{\mathrm{i}}$ is the corresponding partition value of $\Pi(D)$.

In recoding phase of this new algorithm, the canonical recoding is performed on the multiplier. The canonical recoding guarantees the minimal 
Hamming weight. In partitioning phase, the partitioning is performed on the resulted signed-digit multiplier. So the number of zero partitions is as large as possible and the number of multiplication steps is reduced considerably. The CLNZ partitioning strategy instrumented in this algorithm scans the multiplier from the least significant digit to the most significant digit according to the finite state machine shown in Figure 1. In this strategy, zero windows are allowed to have an arbitrary length, but the maximum length of nonzero windows should be the exacted value of $d$ digit.

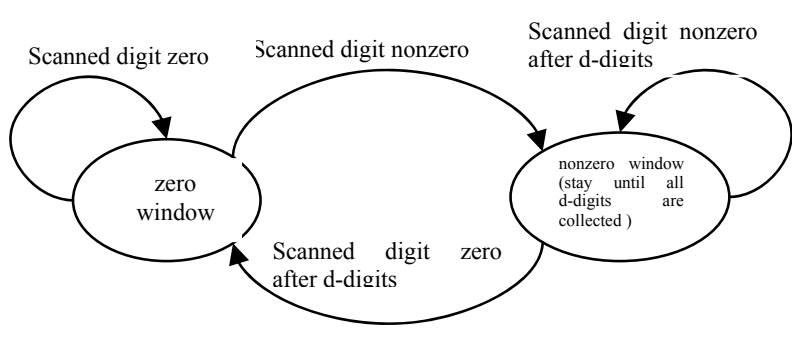

Figure 1. The partitioning strategy

For example, for $\mathrm{X}=(011111110011111110)_{2}$, the canonical recoding of $\mathrm{X}$ is $\mathrm{D}=(1000000101000000 \overline{10})$ and for $\mathrm{d}=3$, the window formed will be $\Pi(D)=(001),(000000),(10 \overline{1}),(00000),(010)$. In this example, there are 14, 4 and 3 nonzero digit in binary representation, canonical representation and proposed representation respectively. Also for $\mathrm{X}=$ $(01011111111001)_{2}$, the canonical recoding of $\mathrm{X}$ is $\mathrm{D}=(010100000001001)$ and for $\mathrm{d}=4$, the window formed will be $\Pi(D)=(010 \overline{1}),(0000000),(1001)$. In this example, there are 10, 4 and 2 nonzero digit in binary representation, canonical representation and proposed representation respectively. From these two examples, we can observe that by increased the window width; the Hamming weight of the number is decreased.

The transition probability graph of proposed modular multiplication is shown in Figure 2. This graph is similar to the transition probability graph of the adaptive m-ary segmentation canonical recoding multiplication algorithm in [13][14].

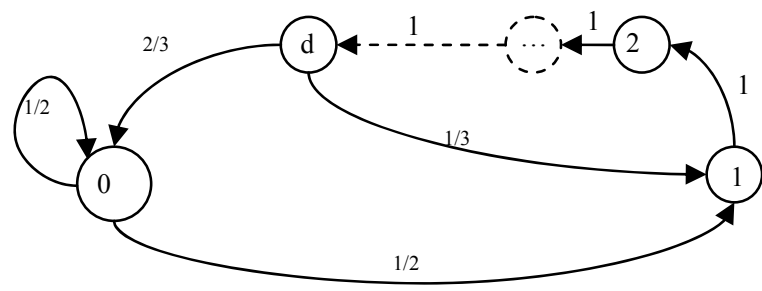

Figure 2. Transition probability graph for the proposed modular multiplication algorithm
In pre-computation phase of algorithm 6, the least significant digit of nonzero partition is either 1 or $\overline{1}$, which implies that the nonzero partition value is always an odd number. So we don't require pre-computation of $\mathrm{V}_{\mathrm{i}} \mathrm{Y}$ for even number of $\mathrm{V}_{\mathrm{i}}$.

Note that the pre-computation phase and the partition phase are performed independently in parallel. This speeds up modular multiplication.

The multiplication phase of algorithm 6 is performed w times. Recall that $\mathrm{w}$ denote the number of partitioning in the signed-digit multiplier. In the each iteration of multiplication phase of algorithm 6, $l_{i}$ bits of multiplier and n-bit multiplicand are processed.

We propose also using this new modular multiplication algorithm in order to speeding up the CMM-MSD Montgomery exponentiation algorithm [17] as shown in algorithm 7.

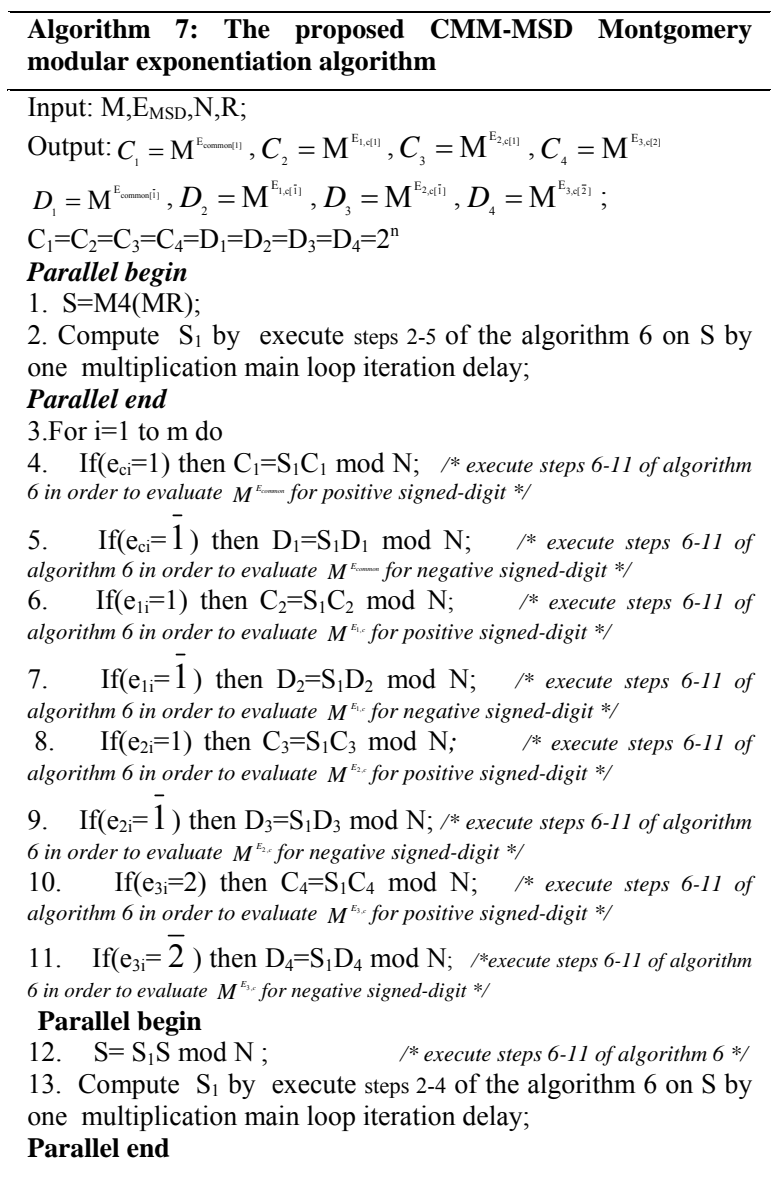

In this algorithm, $\mathrm{E}_{\mathrm{MSD}}=\mathrm{E}_{1}\left\|\mathrm{E}_{2}\right\| \mathrm{E}_{3}$ where $\|$ is the concatenation operator where $\mathrm{E}_{\mathrm{i}}=\mathrm{E}_{\mathrm{i}, \mathrm{c}}+\mathrm{E}_{\text {common. Also }}$ $\mathrm{C}_{1}, \mathrm{C}_{2}, \mathrm{C}_{3}, \mathrm{C}_{4}, \mathrm{D}_{1}, \mathrm{D}_{2}, \mathrm{D}_{3}$ and $\mathrm{D}_{4}$ are eight different registers in order to save intermediate results where $\mathrm{C}_{\mathrm{i}}$ and $\mathrm{D}_{\mathrm{i}}$ are used in order to save positive digit results and negative digit results respectively.

In this new CMM-MSD Montgomery modular exponentiation algorithm, the pre-computation phase 
of algorithm 6 is performed just once in the beginning of the algorithm.

In step 1 of algorithm $7, \mathrm{~S}$ is computed by using algorithm 6 . In step $2, \mathrm{~S} 1$ is computed by executing steps $2-5$ of algorithm 5 on $\mathrm{S}$ by one multiplication loop delay. In steps 4-11 of algorithm 7, the $M^{E_{\text {common }}}, M^{E_{1, c}}, M^{E_{2, c}}$ and $M^{E_{3, c}}$ is computed based on value of the $\mathrm{e}_{\mathrm{ij}}$. These values are computed by executing steps 6-11 of algorithm 6. In steps 12-13 of algorithm 7, the partial result, S, and S1 are computed by executing steps 6-11 and steps 2-4 of algorithm 6 respectively. In this algorithm $\mathrm{S} 1$ is computed by one multiplication loop delay in compare with computation of S. The exponentiation operation ME can be depicted as (1).

\section{Security analyses}

In cryptosystems, while secret data are being processed they can be deduced by observing the execution time, the power consumption and electromagnetic radiation [18]. In the M4 algorithm, multiplication by zero digits with any length is performed in only one cycle instead of several cycles. So attackers can't use knowledge of the difference time and power consume between zero digit and nonzero digit processing. Also in the proposed CMM-MSD Montgomery exponentiation algorithm, the parallel structure is used in all computation stages. So the cryptosystems which use of the proposed CMM-MSD Montgomery exponentiation algorithm is standing against timing analysis attacks and simple power analysis (SPA) attacks. In addition exploitation of the key information by measurement of the currents following through each component of the cryptography device is hard. Thus, the hardware implementation of the cryptosystem, which use of the proposed CMM-MSD Montgomery exponentiation algorithm, is standing against electromagnetic analysis (EMA) attacks. Therefore, the security of the cryptosystems which use of this new CMM-MSD Montgomery exponentiation algorithm increased considerably.

\section{Evaluation}

In the proposed CMM-MSD Montgomery modular exponentiation algorithm, we use radix-3 signed-digit exponent. So the occurrence probability of digits is as following:

$\mathrm{P}(0)=2 / 3, \mathrm{P}(1)=\mathrm{P}(-1)=\mathrm{P}(2)=\mathrm{P}(-2)=1 / 12$.

Therefore based on the computational analyses of Montgomery reduction algorithm from [15] for n-bit modulus and $\mathrm{k}$-bit exponent, the following four operations $\mathrm{S}_{1} \mathrm{C}_{1} \bmod \mathrm{N}, \mathrm{S}_{1} \mathrm{C}_{2} \bmod \mathrm{N}, \mathrm{S}_{1} \mathrm{C}_{3} \bmod \mathrm{N}$ and $\mathrm{S}_{1} \mathrm{C}_{4} \bmod \mathrm{N}$ require
$6 \times \frac{1}{12}\left[1.5 k\left(\frac{6 n}{3 d+4}-2\right)(n+1)\right]=\frac{3}{4} k\left(\frac{6 n}{3 d+4}-2\right)(n+1)$

multiplication steps. Similarly, the following four operations $\mathrm{S}_{1} \mathrm{D}_{1} \bmod \mathrm{N}, \mathrm{S}_{1} \mathrm{D}_{2} \bmod \mathrm{N}, \mathrm{S}_{1} \mathrm{D}_{3} \bmod \mathrm{N}$ and $\mathrm{S}_{1} \mathrm{D}_{4} \bmod \mathrm{N}$ require

$6 \times \frac{1}{12}\left[1.5 k\left(\frac{6 n}{3 d+4}-2\right)(n+1)\right]=\frac{3}{4} k\left(\frac{6 n}{3 d+4}-2\right)(n+1)$

multiplication steps. Also the operation $\mathrm{S}_{1} \mathrm{~S} \bmod \mathrm{N}$ requires

$\frac{2}{3}\left[0.5 k\left(\frac{6 n}{3 d+4}-2\right)(n+1)\right]=\frac{1}{3} k\left(\frac{6 n}{3 d+4}-2\right)(n+1)$

multiplication steps. Therefore the proposed modular exponentiation algorithm takes

$\frac{3}{4} k\left(\frac{6 n}{3 d+4}-2\right)(n+1)+\frac{3}{4} k\left(\frac{6 n}{3 d+4}-2\right)(n+1)+\frac{1}{3} k\left(\frac{6 n}{3 d+4}-2\right)(n+1)$

$=1.833 k\left(\frac{6 n}{3 d+4}-2\right)(n+1)$

multiplication steps, however the adopted Montgomery modular reduction (MMR) algorithm [2], the Ha-Moon's improved Montgomery algorithm [15], the $\mathrm{Wu}$ et al.'s CMM-MSD algorithm [16] and Wu's improved CMM-MSD algorithm [17] require $1.5 k\left(2 n^{2}+n\right)$, $0.5 k\left(5 n^{2}+4 n\right), \quad 0.5 k\left(2 n^{2}+2 n+0.75\right) \quad$ and $1.833 \mathrm{k}\left(\mathrm{n}^{2}-\mathrm{n}-2\right)$ multiplication steps respectively. On average, the proposed modular exponentiation algorithm reduces the overall number of multiplication steps at about

$$
\begin{aligned}
& 1-\frac{1.833 k\left(\frac{6 n}{3 d+4}-2\right)(n+1)}{1.5 k\left(2 n^{2}+n\right)} \approx 1-\frac{11}{3(3 d+4)} \\
& 1-\frac{1.833 k\left(\frac{6 n}{3 d+4}-2\right)(n+1)}{0.5 k\left(5 n^{2}+4 n\right)} \approx 1-\frac{11}{2.5(3 d+4)} \\
& 1-\frac{1.833 k\left(\frac{6 n}{3 d+4}-2\right)(n+1)}{1.833 k\left(n^{2}-n-2\right)} \approx 1-\frac{11}{1.833(3 d+4)} \\
& 1-\frac{1.833 k\left(\frac{6 n}{3 d+4}-2\right)(n+1)}{0.5 k\left(2 n^{2}+2 n+0.75\right)} \approx 1-\frac{11}{(3 d+4)}
\end{aligned}
$$

in compare with [2][15][17][16] respectively.

We summarize the multiplication steps improvement for the proposed CMM-MSD Montgomery modular exponentiation algorithm over exponentiation algorithm in [2][15][16][17] for various window width in table 1 .

Table 1. Multiplication step improvement of the proposed CMMMSD Montgomery modular exponentiation algorithm

\begin{tabular}{|c|c|c|c|c|}
\hline \multirow{2}{*}{$\begin{array}{c}\text { Window } \\
\text { width }\end{array}$} & \multicolumn{4}{|c|}{ Improvement percentage } \\
\cline { 2 - 5 } & {$[\mathbf{2}]$} & {$[\mathbf{1 5}]$} & {$[\mathbf{1 7 ]}$} & {$[\mathbf{1 6}]$} \\
\hline $\boldsymbol{d}=\mathbf{3}$ & $71.8 \%$ & $66.2 \%$ & $53.8 \%$ & $15.4 \%$ \\
\hline $\boldsymbol{d}=\mathbf{4}$ & $77 \%$ & $72.5 \%$ & $62.5 \%$ & $31.3 \%$ \\
\hline $\boldsymbol{d}=\mathbf{5}$ & $80.7 \%$ & $76.8 \%$ & $68.4 \%$ & $42.1 \%$ \\
\hline
\end{tabular}




\begin{tabular}{|c|c|c|c|c|}
\hline $\boldsymbol{d}=\mathbf{6}$ & $83.3 \%$ & $80 \%$ & $72.7 \%$ & $50 \%$ \\
\hline $\boldsymbol{d}=\mathbf{7}$ & $85.3 \%$ & $82.4 \%$ & $76 \%$ & $56 \%$ \\
\hline $\boldsymbol{d}=\mathbf{8}$ & $86.9 \%$ & $84.3 \%$ & $78.6 \%$ & $60.7 \%$ \\
\hline $\boldsymbol{d}=\mathbf{9}$ & $88.2 \%$ & $85.8 \%$ & $80.6 \%$ & $64.5 \%$ \\
\hline $\boldsymbol{d}=\mathbf{1 0}$ & $89.2 \%$ & $87.1 \%$ & $82.3 \%$ & $67.6 \%$ \\
\hline
\end{tabular}

These results are represented graphically in Figure 3.

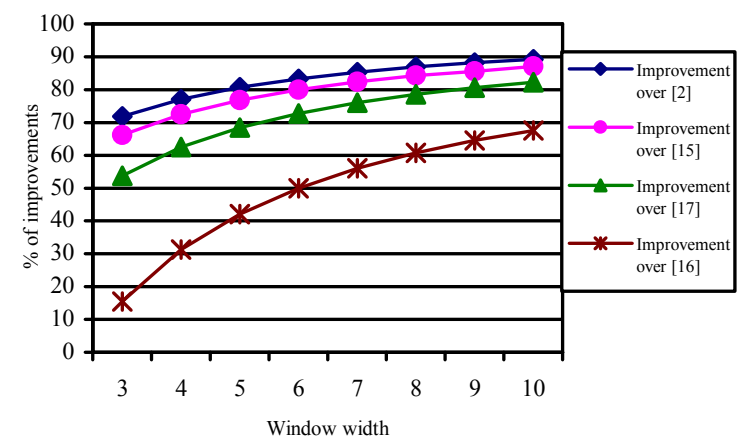

Figure 3. Multiplication step improvement of the proposed CMM-MSD Montgomery modular exponentaition

As it is shown in Table 1 and Figure 3, the proposed modular exponentiation algorithm reduces the multiplication steps considerably.

The results show that this new modified CMM-MSD Montgomery exponentiation algorithm reduces on average the number of multiplication steps at about $71.8 \%-89.2 \%, 66.2 \%-87.1 \%, 15.4 \%$ $67.6 \%$ and $53.8 \%-82.3 \%$ in compare with DusseKaliski's Montgomery algorithm [2], Ha-Moon's Montgomery algorithm [15], Wu et al.'s CMM-MSD Montgomery algorithm [16] and Wu's improved CMM-MSD Montgomery algorithm respectively for $\mathrm{d}=3-10$.

\section{Conclusion}

In this paper, we present a new efficient CMM-MSD Montgomery exponentiation algorithm based on the modified Montgomery modular multiplication. In addition this new modular exponentiation algorithm uses other techniques such as: minimal signed-digit (MSD) recoding, sliding window method and common-multiplicand-multiplication $\quad(\mathrm{CMM})$ method. By performing the MSD recoding technique on multiplier, the probability of the zero bits is increased. Furthermore by performing the sliding window method on signed-digit multiplier, the multiplication steps are reduced considerably. The modified Montgomery modular multiplication is also skipped from zero digit multiplication and is shifted in only one cycle by using Barrel shifter instead of several cycles. By using CMM method, the common part of the modular multiplication is computed once rather than several times. So the security of the cryptosystem which used this new algorithm increased considerably. The results show that the number of multiplication steps in the proposed
CMM-MSD Montgomery exponentiation algorithm is reduced on average at about $71.8 \%-89.2 \%$, $66.2 \%-87.1 \%, 15.4 \%-67.6 \%$ and $53.8 \%-82.3 \%$ in compare with Dusse-Kaliski's Montgomery algorithm [2], Ha-Moon's Montgomery algorithm [15],Wu et al.'s CMM-MSD Montgomery algorithm [16] and Wu's improved CMM-MSD Montgomery algorithm [17] respectively for $\mathrm{d}=3-10$.

\section{References}

[1] N. Nedjah and L.M. Mouller, "Hihgh-performance hardware of the sliding-window method for parallel computation of modular exponentitions," international journal of parallel programming, Springer Netherlands, vol.37, pp.537-555, 2009.

[2] S. R. Dusse, B. S. Kaliski, “A cryptographic library for the Motorola DSP 56000," Advance in Cryptology Proceedings of UROCRYPT'90, LNCS, vol.73, pp. 230-244, 1990.

[3] P. Keshavarzi and C. Harrison, "A new modular multiplication algorithm for VLSI implementation of public-key cryptography," Proceedings of First International Symposium on Communication Systems and Digital Signal Processin, pp.516-519, 1998.

[4] K. Sakiyama, L. Batina, B. Preneel and I. Verbauwhede, " High-performance publik-key cryptoprocessor for wireless mobile applications," Mobile networks and applications, vol. 99, pp. 245-258, 2007.

[5] A. Rezai and P. Keshavarzi, "Improvement of highspeed modular exponentiation algorithm by optimum using smart methods, " Proceedings of 18th Iranian Conference on Electrical Engineering, Iran, pp.2104-2109, May 2010.

[6] A. Rezai and P. Keshavarzi, "Speed Improvement in elliptic curve cryptosystem scalar multiplication algorithm," proceedings of 7th International ISC Conference on Information Security and Cryptology2010, Iran, pp.181-188, September 2010.

[7] P. L. Montgomery, "Modular multiplication without trial division", Mathematics of computation, vol. 44, no. 170 , pp. 519-521,1985.

[8] A.F.Tenca and C.K.Koc, "A scalable architecture for modular multiplication based on Montgomery's algorithm,'IEEE Trans. On computer, vol.52, no.9, pp. 1215-1221, 2003.

[9] N.Pinckney, P. Amberg and D. Harris, "Parallelized Booth-encoded radix-4 Montgomery multipliers," proceeding of 16th IFIP/IEEE Intlernational Conferene on Very Large Scale Integration, Oct. 2008.

[10] T. Blum and C. Paar, "High-radix Montgomery multiplication on reconfigurable hardware," IEEE Trans. on computers, vol. 50, no.7 pp. 759-764, 2001.

[11] N. Pinckney and D. Harris, "Parallelized radix-4 scalable Montgomery multipliers," Journal of Integrated Circuits and Systems, vol.3, no.1, pp. 39-45,2008. 
[12] L. A. Tawalbeh, A. F. Tenca, and C. K. Koc, “A radix4 scalable design," IEEE Potentials,vol.24, no.2, pp.16 - 18, 2005.

[13] C.K.Koc and C.Y.Hung, "Adaptive m-ary segmentation and canonical recoding algorithms for multiplication of large binary numbers," Computer mathematic application, vol.24, no.3, pp.3-12, 1992.

[14] B.Philips and N.Burgess, "Minimal weight digit set conversions," IEEE Trans. on computers, vol. 53, no.6 pp. 666-677, 2004.

[15] J.C. Ha, S.J. Moon, “A common-multiplicand method to the Montgomery algorithm for speeding up exponentiation," Information Processing Letters, vol.66, no.2, pp.105-107,1998.

[16] C.Wu, D.Lou and T.Chang, "An efficient Montgomery exponentiation algorithm for public-key cryptosystem," Proceedings of IEEE international conference on intelligence and security information, pp.284-285, June 2008.

[17] C.Wu, "An efficient common-multiplicandmultiplication method to the Montgomery algorithm for speeding up exponentiation," Information Sciences, vol.179, pp.410-421, 2009.

[18] L. Batina, et al. "Side channel attacks and fault attacks on cryptographic algorithm," Revue HF Tijdschrift vol. 3, pp. 36-45, 2004. 